after DNA damage. A model that accommodates these apparently conflicting observations is outlined in Fig. 2. Perhaps the function of SUMO is to allow NEMO to be incorporated into an ATM signalling complex. Once incorporated into the complex, SUMO is no longer required and can be removed by the action of an isopeptidase, leaving the now unmodified NEMO locked in to the signalling scaffold. After phosphorylation of NEMO induced by ATM, the role of ubiquitin could be to release the phosphorylated NEMO from the complex. Once NEMO has been released, the ubiquitin is no longer required and can be removed by one of the large family of ubiquitin isopeptidases. This model would explain why the lysine residues are absolutely required for DNA damage signalling and why only tiny amounts of SUMO- and ubiquitin-modified species are ever detected. Although no genetic or siRNA experiments are presented that demonstrate a role for SUMO or ubiquitin in DNA damage signalling to $\mathrm{NF}-\kappa \mathrm{B}$, it is likely that these experiments will be forthcoming. Assuming that this is indeed the case, then these pathways may well become targets for the development of anti-cancer drugs. If these modifications have a general role in the DNA damage response, as yeast genetic experiments suggest, then their inhibition could enhance cancer therapy by both inhibiting the DNA repair process and the survival response mediated by NF-кB.

1. Huang, T. T., Wuerzberger-Davis, S. M., Wu, Z. H. \& Miyamoto, S. Cell 115, 565-576 (2003).

2. Ghosh, S. \& Karin, M. Cell 109, S81-S96 (2002).

3. Huang, T. T., Feinberg, S. L., Suryanarayanan, S. \& Miyamoto, S. Mol. Cell Biol. 22, 5813-5825 (2002).

4. Doffinger, R. et al. Nature Genet. 27, 277-285 (2001).

5. Jain, A. et al. Nature Immunol. 2, 223-228 (2001).

6. Rodriguez, M. S., Dargemont, C. \& Hay, R. T. J Biol Chem 276, 12654-12659 (2001).

7. Li, N. et al. J. Biol. Chem. 276, 8898-8903 (2001).

8. Piret, B., Schoonbroodt, S. \& Piette, J. Oncogene 18, 2261-2271 (1999).

9. Seeler, J. S. \& Dejean, A. Nature Rev. Mol. Cell Biol. 4, 690-699 (2003).

10. Desterro, J. M., Rodriguez, M. S. \& Hay, R. T. Mol. Cell 2, 233-239 (1998).

11. Hoege, C., Pfander, B., Moldovan, G. L., Pyrowolakis, G. \& Jentsch, S. Nature 419, 135-141 (2002).

\section{Security measures at the nuclear pore}

At each stage of the cell cycle, it is crucial that certain cell-cycle regulators enter the nucleus while others are excluded. The nuclear pore complex (NPC) is the gateway between the nucleus and the cytoplasm; nucleo-cytoplasmic transport is mediated by import and export receptors, which bind diverse sets of cargo and mediate their movement through the pore by regulated interactions with its nuclear pore protein (nucleoporin) scaffold. Although previous studies of transport during mitosis have largely highlighted how post-translational modifications of the cell-cycle machinery can alter their interactions with the nuclear transport machinery, and thus their localization, new work from Rick Wozniak and colleagues (Cel/ 115, 813-823; 2003) identifies an additional layer of control - rearrangement of the nuclear pore complex itself.

The authors began with the observation that mitosis is delayed in mutants of the yeast import receptor Kap121p. One explanation for this is that controlling Kap121p-mediated transport is important for mitotic progression; consistently, they find that Kap121p cargoes are no longer imported into the nucleus during mitosis. This did not seem to be caused by altered binding of cargo to Kap121p, but rather because import of Kap121p is specifically inhibited during mitosis.

One explanation for reduced Kap121p import is through altered interactions with the NPC. As Kap121p interacts specifically with Nup53p, the authors asked whether this interaction is cell-cycleregulated. Indeed, they found that Nup53p binds to Kap121p only during G2/M. This interaction is driven by a number of specific molecular rearrangements in the NPC that alter the interactions of neighbouring nucleoporins with Nup53p and expose a binding site for Kap121p. Association with Nup53p blocks movement of Kap121p through the NPC and, at least in vitro, triggers the release of cargo from Kap121p.

Consistently, overexpression of Nup53p inhibits transport of Kap121p, resulting in similar mitotic phenotypes to kap121 mutants. Moreover, from this inhibitory model one would predict that disrupting this interaction would prolong import of Kap121p

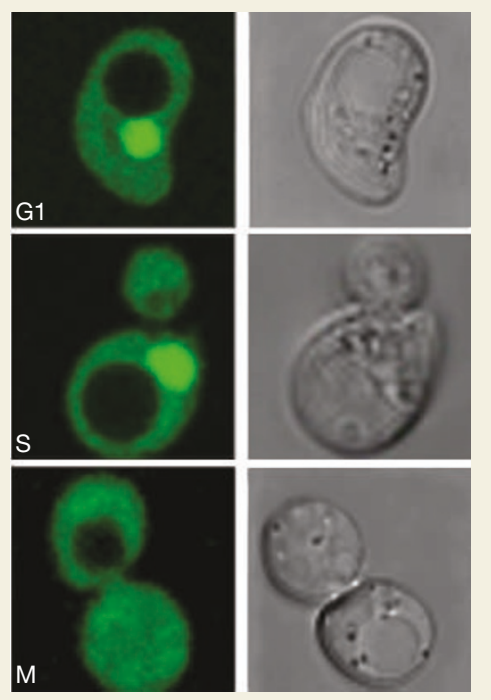

Kap121p-mediated nuclear import in inhibited during mitosis. Fluorescence and phase-contrast images of yeast cells expressing a green fluorescent protein (GFP)-tagged Kap121p cargo and arrested in $\mathrm{G} 1, \mathrm{~S}$ and $\mathrm{M}$ phase. Import is inhibited in the mitotic cell. The circular regions lacking GFP in each cell are the vacuoles.

during $\mathrm{G} 2 / \mathrm{M}$; this is indeed what they see. So, taken together, Wozniak and colleagues propose a model in which as yet undefined signals orchestrate changes in the NPC that allow Nup53p to function as a transport-inhibitory nucleoporin, a previously unforeseen function for a nucleoporin.

It will be interesting to see whether other rearrangements are also occurring at the pore during mitosis, and whether this mechanism also applies to other transport receptors and, potentially, to other points in the cell cycle. It will, of course, be important to learn which cargos of Kap121p are responsible for the mitotic defects observed when this mechanism is disrupted. Finding the answers will be a challenge, given that Kap121p is known to have multiple cargos and that import is often mediated by redundant transport receptors.

ALISON SCHULDT 